\title{
A Modern themed System for Patients Security of data exposure in semi-convinced Servers in the Cloud
}

\author{
Parikshith Nayaka S K ${ }^{1}$, Dayanand Lal. ${ }^{2}$, Vasudev Shahapur ${ }^{3}$, Saritha A K $\mathrm{K}^{4}$, Nida Kousar ${ }^{5}$ \\ ${ }^{1}$ Assistant Professor, Department of CSE, GITAM School of Technology, Bengaluru, India, pari2sn @ gmail.com \\ ${ }^{2}$ Assistant Professor, Department of CSE, GITAM School of Technology, Bengaluru, India, \\ dayanandlal@gmail.com \\ ${ }^{3}$ Associate Professor, Department of CSE, Alva's Institute of Technology, Moodbidri, India, \\ shahapurvasu@gmail.com \\ ${ }^{4}$ Assistant Professor, Department of CSE, GITAM School of Technology, Bengaluru, India, savikkal@ gitam.edu \\ ${ }^{5}$ Assistant Professor, Department of CSE, GITAM School of Technology, Bengaluru, India, knida@ gitam.edu
}

\begin{abstract}
Cloud computing is intermingled distributed storing platform where information is housed in virtualized storage collections which are normally operated by tertiary parties. Patients healthiness record (PHR) is an evolving patient-centered paradigm of healthiness info sharing, problems such as threats of confidentiality leakage, key management scalability, scalable access, and effective consumer revocation, provide the most significant challenges if we introduce a modern patient-centered architecture and a series of info contact control protocols for PHRs maintained in semi-trusted repositories. They use attribute-based encryption (ABE) strategies to encrypt the PHR file of each individual to obtain fine-grained and robust user access protection for PHR's. The project often provisions numerous owner situations and splits the device workers into many protection fields which significantly reduces the difficulty of key managing for holders and handlers. We introduce this program and test upon Drive HQ cloud to validate that our latest framework offers safe data control to outsourced info.
\end{abstract}

Key words: Cloud storage, attribute-based encryption and personal health data.

\section{INTRODUCTION}

Cloud hosting is already the scorching advert for computer company and science, as it gives clients an extension to unlimited cloud capacity to store information tie-ups in a pay-as-you-go method. It allows corporations and administration departments to substantially lessen their overheads. While they will now store their information tie-ups on third-party cloud service services directly instead of running data centers of their own. Recent years have seen the rise of personal health record (PHR) as a patient-centered platform for sharing healthiness records. A PHR program helps a patient to build, monitor and track their personal health records in one location across the internet, allowing it more convenient to store, access and exchange medical detailsIn fact, a individual is given absolute autonomy of their medical history and can exchange their health details with a broad variety of people including health care professionals, family members or associates. Most PHR roles are farm out to third-party software dealers, such as Microsoft HealthVault, owing to the high expense of constructing and managing complex data centres. Cloud storage systems for processing PHRs were introduced in [2], [3]

Although providing easy PHR facilities for everybody is exciting, there are also protection and privacy threats that could hinder its wide-ranging acceptance. The key issue is that patients may effectively monitor the exchange of their confidential individual health details (PHI), particularly if they are housed on a third-party website that public do not faith entirely. On one side, while here are health care laws such as HIPAA, which has recently been revised to include professional links, cloud services are typically not protected [13]. At the other side, the third-party database systems are also the objects of different fraudulent activities owing to the high importance of the confidential PHI, which may contribute to PHI leakage. This is important to provide fine-grained data access management systems that operate with semi-trusted providers to maintain patient-centered confidentiality rights over their personal PHR's.

Crypting the data before outsourcing will be a viable and successful solution. The PHR owner will basically determine whether to encode his / her data and require which group of users to get access to could information. Only users who are provided the accompanying decryption key will have access to a PHR register, thus staying private to the other users. In addition, the patient must also maintain the exact not individual to contribution but also to retract admittance 
privileges if they believe it is appropriate [7]. Nonetheless, in a PHR framework, the objective of patient-centered privacy is always at odds with the scalability. The approved users can need to use the PHR either for personal usage or for technical use. Sources are family members and associates, whereas the latter may be clinicians, pharmacists, and scholars, etc. The two types of users are referred to separately as informal and technical users. The above is theoretically high in scale; should each owner be specifically liable for handling all the skilled customers, the main management overhead would quickly overtake her, determining a list of these is complicated for the user. In the other hand, in a PHR scheme, different from the sole information owner situation found in utmost of the current works[8],[9], there are several owners who can encrypt through their own means, probably utilizing various sets of cryptographic keys. Allowing every consumer to get solutions from any owner who is PHR that she needs to speak will restrict usability, because pat ients are not all available. An option is to appoint a chief expert (CA) to administer significant for all PHR members, but this involves too ample trust in a sole specialist (i.e., origin the crucial escrow problematic).

In this article, we are working to research the patient-centered, safe exchange of PHRs held on semi-trusted host and concentrate on solving the difficult and daunting issues of significant organization. We accept attribute-based encryption $(\mathrm{ABE})$ as the primitive principal encryption to guard the personal health information stowed on a semi-trusted host. By means of ABE, admittance strategies are represented on the basis of user attributes or files, which allows a patient to collectively distribute their PHR with a group of handlers by encoding the file underneath a set of features, without needing to learn a full user list. The complications of encoding, key creation, and decoding are only linear due to the amount of qualities complicated. Nonetheless, to incorporate ABE into a important PHR program, critical matters such as core administration scalability, complex regulation changes and effective revocation of on-demand are not easy to address, and remain mostly UpToDate. Toward that end, we are making the subsequent major aids:

We suggest a new, patient-centered, safe collaboration system with PHRs cloud computing settings. User will delegate the significant to the person he / she wants to pass the file to. We left habit to give the key to tackle the key management challenges. The bulk of skilled users in particular are handled by rating, although each controller only needs to control the keys of a limited amount of users in their specific area as well. In this mode, our architecture will meet the demands of various forms of PHR sharing applications concurrently, thus incurring reduced overhead of key administration for both device owners and consumers.
However, the system guarantees fewer effort to provide write access control, performs complex regulation changes, and offers break-glass exposure to PHRs during emergence situations by supplying exposure to the attribute with the support of the Protection authority.

Owners explicitly grant access rights to individual users and encode a PHR file under their information characteristics, and under normal protection assumptions show their health. By this way patients have full power of their PHRs by terms of safety. In terms of several measurements in computing, connectivity, storage and key management, we offer a detailed overview of the functionality and determinate of our planned safe PHR sharing approach. In terms of complexity, scalability and security we equate our system with some earlier schemes too. In addition, we validate the effectiveness of our structure by applying it on a modern terminal and carrying out researches.

\section{LITERATURE SURVEY}

Partially trustworthy servers are also presumed for access control of the outsourced data. Using cryptographic methods, the purpose is to determine who has (read) exposure in a fine-grained fashion to certain sections of a patient's PHR records.

\section{Symmetric key crypto-centred solution:}

Symmetric key systems are a session of cryptographic processes which use the similar crypto key for both plaintexts encoding then cryptograph text decoding. The solutions may be the same or between the two keys there might be a easy transition to go. The keys reflect, in fact, a mutual top-secret amongst two or extra revelries that tin be castoff to preserve a sequestered knowledge connection Vimercati et.al.[6] Suggested a key to safe subcontracted details on semi-trusted hosts focused on symmetric key origin techniques, which can accomplish finegrained access controller. The dynamics of file formation and user contribution / withdrawal actions are sadly proportional to the quantity of official users, which is fewer accessible.

\section{Public key crypto-centred solution:}

Because of its capacity to distinguish write and read rights, PKC related approaches were introduced. The standard public key encryption (PKE) related systems introduced by $\mathrm{J}$ are intended to attain satisfactory grained entree control. Benaloh, Chase M., Horvitz E., K. In their research "Patient-controlled encryption: preserving the protection of electronic medical information," Lauter[8] discusses the approach example which illustrates how transparent which symmetric encryption is used, the drawback of their method is 
either high overhead key storage, which allows several copies of a file to be authenticated with separate user keys.

\section{Attribute Based Encoding centred solutions:}

In particular, a amount of everything used $\mathrm{ABE}$ to perform fine-grained entree regulator for subcontracted information; there was a growing attention in put on $\mathrm{ABE}$ to safe electric healthcare records (EHRs). Narayan et al . suggested an attribute-based architecture for EHR schemes, wherever each patient's EHR data are authenticated using a Encryption Text-ABE (CP-ABE) transmitted variant[4]. Furthermore, the document duration of the cipher decreases linearly with the amount of users. A version of ABE allowing the allocation of entree privileges for authenticated EHRs is introduced. Ibraimi et, etc. [5] the encryption text regulation $\mathrm{ABE}$ (CP-ABE)[11] extended to control PHR sharing; and implemented the idea of societal / expert realms but do not habit multi-authority ABE In[3], Akinyele et al . examined by means of $\mathrm{ABE}$ to create self-protected EMRs that can also be placed on cloud storage or mobile phones such that EMR may be read while the healthcare earner is disconnected. Problem is system reliance which is not provided with the revocation. Another Popular downside of both of the above approaches is the key-escrow issue, since they find a single trustworthy authority.

\section{PROPOSED SYSTEM}

We contemplate a PHR structure with many proprietors of PHR and PHR handlers. Patients who have direct autonomy of their own PHR data are responded to by the holder, i.e. they may build, maintain, and erase it. Here is a chief server that belongs to the PHR provision worker which supplies the PHRs of all the owners. The consumers tin originate since various features; a virtual, caregiver, or investigator, for example. Consumers view the PHR information via the portal and deliver or carve to somebody's PHR, so a person may view several proprietary files at the same time.

In this article, we contemplate the semi-trusting server, implying that server should seek to figure obtainable as ample hidden knowledge as possible in the encrypted PHR data, but they will obey the protocol in general in an honest fashion. At the other side, certain people may even want to move outside their rights to enter the files. A pharmacy, for example, might want to get patient prescriptions for promotion and enhancing its revenues. They can scheme with added users, or uniform the server, in order to do so. Further, we presume that each participant in our program is preloaded with a community / isolated pair, so conventional challenge-response protocols may be used to authenticate the individual.
Fig.1 demonstrates the planned framework design for safe exchange of personal Health information. The network is divided into two protection areas, specifically community domains (PUDs) and private domains (PSDs) rendering to the information contact criteria of the specific users. The PUDs comprise of consumers who render contact dependent on their specific positions, such as surgeons, nurtures, medicinal professionals and coverage officers. Its consumers are directly affiliated through a information proprietor (such as household members or near friends) with each PSD and allows access to health information dependent on the owner's delegated access rights. There we find the data owner who has the medical report, the data user who is able to interpret the medical record authenticated. The possessor cast-off key-policy accredited based encoding in PSD and created undisclosed key for their PSD operators and favored the multi-authority dependent encryption feature in PUD. Different authority creates Hidden Key for PUD consumers, based on their specialization and occupation in combination.

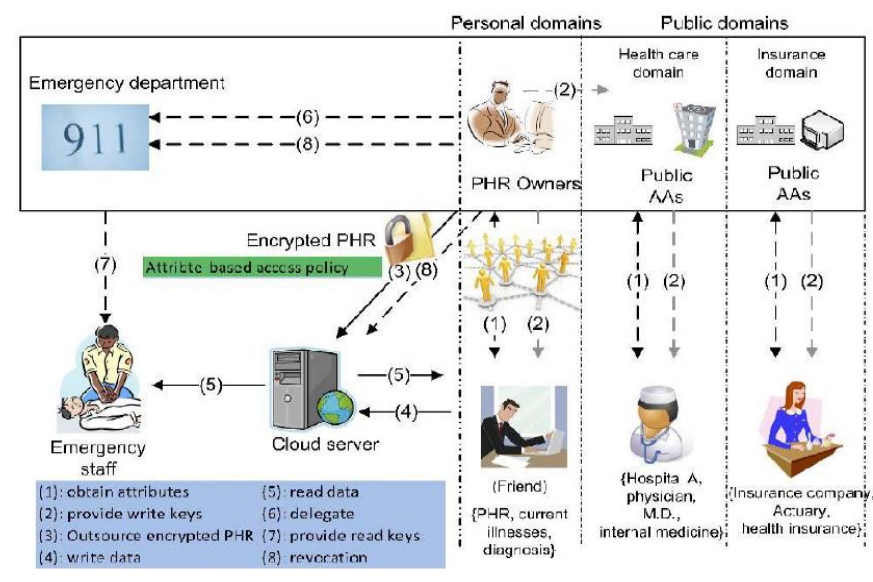

Figure 1: The suggested patient-centered system for secure PHR sharing

\section{ABE System}

This is a kind of community encryption key that depends on the attributes of a user's public key and cryptograph text. There are four steps to a (key policy) quality-based encryption system.

System Features: This process is used to define user qualities. This is a random system which does not take input but the implied parameter for protection. It describes a bilinear collection G1 with the first direction $\mathrm{p}$ through a producer $\mathrm{g}$, and bilinear map e: G1 alternatively G1 alternatively G2 with the belongings of bi-linearity, degeneration and non-degeneration. Public key and master key can be calculated from these qualities for each user. The public key and master key attributes are referred to as

Attributes- $\mathrm{U}=\{1,2.3 .4 \ldots \mathrm{N}\}$

Community key $-\mathrm{P}_{\mathrm{K}}=(\mathrm{Y}, \mathrm{T} 1, \mathrm{~T} 2, \mathrm{~T} 3 . \mathrm{T} 4 ., \ldots \mathrm{T} \mathrm{N})$

Principal key-MK $=(y, t$ 1, t2, t3.t4 ..., t N $)$ 
Parikshith Nayaka S K et al., International Journal of Emerging Trends in Engineering Research, 8(8), August 2020, 4123 - 4127

Notations:

TI $\in$ G1 besides ti $\in \mathrm{Zp}$ stay aimed at quality $\mathrm{i}, 1 \leq \mathrm{i} \leq \mathrm{N}$, in addition $\mathrm{Y} \in \mathrm{G} 2$ is additional community key module. We take $\mathrm{T}$ i=gti too $\mathrm{Y}=\mathrm{e}(\mathrm{g}, \mathrm{g}) \mathrm{y}, \mathrm{y} \in \mathrm{Zp}$. Though $\mathrm{P}_{\mathrm{K}}$ is widely identified to entirely the gatherings in the scheme, MK is reserved as a undisclosed by the specialist party.

Encoding: is a random procedure which takes as input a note $\mathrm{M}$, the public key PK, and a traditional of features I. The cryptograph text $\mathrm{E}$ is output with the arrangement of:

\section{$\mathbf{E}=(\mathbf{I}, \tilde{\mathbf{E}},\{\mathbf{E}$ i $\in \mathbf{I})$}

Notation $\tilde{E}=\mathrm{M}_{\mathrm{Y}}, \mathrm{E} \mathrm{i}=\mathrm{T}$ is. besides $\mathrm{s}$ is haphazardly selected since Z.

Clandestine key generation: is a make random procedure, which takes the admission tree $\mathrm{T}, \mathrm{MK}$, and $\mathrm{K}$ as the input. This gives the following secret user key SK. For each node I of $\mathrm{T}$ it defines first a random polynomial $\mathrm{pi}(\mathrm{x})$ from the root node $r . \operatorname{Pj}(0)=p$ parent $(j)(i d x(j))$ is a unique index of each non- root node $\mathrm{j}$ wherever parental $(\mathrm{j})$ represents a parent $\mathrm{j}$ and iden $x(j)$ is the parent's unique. $\operatorname{Pr}(0)=y$ for the root node $r$. Then SK is produced like this.

\section{SK $=\{$ sk i $\} \mathbf{i} \in \mathbf{L}$}

Wherever $\mathrm{L}$ means the usual of qualities involved to the frond bulges of $\mathrm{T}$ then sk $\mathrm{i}=\mathrm{g}$ pi($(0) / \mathrm{ti}$.

Decoding: The cryptograph text, E, encoded under I, user secret key SK and public key PK are used as an algorithm. Input is the ciphers E. Data. For the leaf nodes, it initially calculates $e(i$, sk $i)=e(g, g) p i(0)$. Then the polynomial interpolation technique aggregates these pairing results in the bottom up way. Finally the shade aspect $\mathrm{Ys}=\mathrm{e}(\mathrm{g}$, g) ys can be recovered and the note $\mathrm{M}$ can be generated only if $\mathrm{I}$ meet $\mathrm{T}$.

\section{RESULTS}

The experimentation tests the various time differences with many Net influences. It takes more than $512 \mathrm{Kbps}$ for us to vary internet link speed as we slowly increase the file size, and it takes less time for $1 \mathrm{Mbps}$ internet connection speed compared to the first. So this displays a lined arc by way of gradually increase the file size and at last it takes less time for a $2 \mathrm{Mbps}$ internet connection speed as compared with the above two in the table.

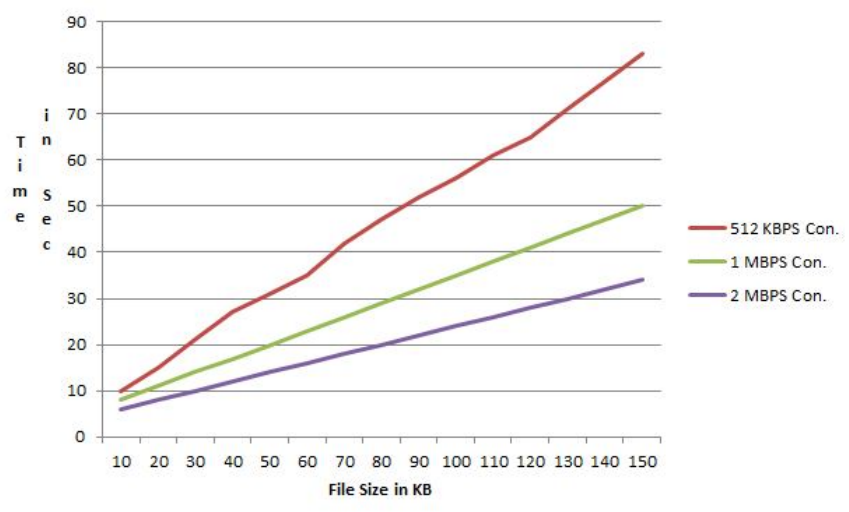

Figure 2: Time Analysis of specific speed of Internet connection

\section{CONCLUSION}

In this article, a novel system for safe storage of private healthiness data in cloud computing has been suggested. The central slogan of the patient typical is to share the patient's personal healthiness accounts with the utmost security, because the cloud servers are reliable. In order to enable fine grain entry, patients shall have complete control over the security of PHR data. In order to allow patients access by not just their private users, then similarly specific users in public networks with various qualified positions, credentials and associations, we encode the PHR records built on the system ABE (attribute dependent encryption). In addition, we are improving an established MA-ABE system to manage app revocation effectively and on requests. We demonstrate our solution is both scalable and efficient through implementation. We will add Video File and User Maintaining documents in our Future Work that we all retrieved the Data.

\section{REFERENCES}

1. M.Li,S. Yu, K. Ren, and W.Lou, "Securing Personal Health Records in Cloud Computing: Patient- Centric and Fine-Grained Data Access Control in Multi-Owner Settings," pp. 89-106, Sept. 2010

2. H. Lo" hr, A.-R. Sadeghi, and M. Winandy, "Securing the E-Health Cloud," pp. 220-229, 2010.

3. A. Akinyele, C.U. Lehmann, M.D. Green, M.W. Pagano, Z.N.J. Peterson, and A.D. Rubin. Self-protecting electronic medical records usingattribute-based encryption on mobile device. Technical report, Cryptology ePrint Archive, Report 2010/565, 2010.

4. S. Narayan, M. Gagn'e, and R. Safavi-Naini, "Privacy preserving ehr system using attribute-based infrastructure," ser. CCSW '10, 2010, pp. 47-52. https://doi.org/10.1145/1866835.1866845

5. L. Ibraimi, M. Petkovic, S. Nikova, P. Hartel, and W. Jonker, "Ciphertext-policy attribute-based threshold 
Parikshith Nayaka S K et al., International Journal of Emerging Trends in Engineering Research, 8(8), August 2020, 4123 - 4127

decryption with flexible delegation and revocation of user attributes," 2009.

6. "At Risk of Exposure - in the Push for Electronic Medical Records, Concern Is Growing About How Well Privacy Can Be Safeguarded, Jun 26,2006.

7. K.D. Mandl, P. Szolovits, and I.S. Kohane, "Public Standards and Patients' Control: How to Keep Electronic Medical Records Accessible but Private," BMJ, vol. 7281, pp. 283-287, Feb. 2001.

8. J. Benaloh, M. Chase, E.Horvitz, and K. Lauter, "Patient Controlled Encryption: Ensuring Privacy of Electronic Medical Records," pp. 103-114, 2009. https://doi.org/10.1145/1655008.1655024

9. S. Yu, C. Wang, K. Ren, and W. Lou, "Achieving Secure, Scalable, and Fine-Grained Data Access Control in Cloud Computing," Proc. IEEE INFOCOM '10, 2010.

10. S. Yu, C. Wang, K. Ren, and W. Lou, "Attribute Based Data Sharing with Attribute Revocation," (ASIACCS '10), 2010

11. J. Bethencourt, A. Sahai, and B. Waters, "Ciphertext-Policy Attribute-Based Encryption" 2007.

12. J. Hur and D.K. Noh, "Attribute-Based Access Control with Efficient Revocation in Data Outsourcing Systems," vol. 22, no. 7, pp. 1214-1221, July 2011.

13. "The Health Insurance Portability and Accountability Act,",

http://www.cms.hhs.gov/HIPAAGenInfo/01_Overview. asp, 2012

14. Mr. Parikshith Nayaka S K, Mrs. Shobha Rani, Dr. Dayanand Lal, Dr. M Anand. (2020). Convert Channel and Information Hiding in TCP/IP . International Journal of Control and Automation, 13(02), 582 - 591. Retrieved from http://sersc.org/journals/index.php/IJCA/article/view/11 199

15. Jacob, I. Jeena. (2020). Ensuring Network Security using Secured Privileged Accounts. International Journal of Emerging Trends in Engineering Research. 8. 1959-1963. 10.30534/ijeter/2020/80852020. https://doi.org/10.30534/ijeter/2020/80852020

16. Fuzzy logic based proportional integral control of frequency for small, International Journal of Advanced Trends in Computer Science and Engineering, 2020, volume 9, number 2, pages 1275-1279 Ramaswamy, K. and Dayanand Lal, N. and Parikshith Nayaka, S.K. and Venna, R.C. and Brahmananda, S.H https://doi.org/10.30534/ijatcse/2020/57922020

17. Effective and Secure Approach for Multi-Keyword Quest Graded over Authenticated Data, International Journal of Advanced Trends in Computer Science and Engineering, 2020, volume 9, number 2, pages 2278-3091, Shobharani D, Parikshith Nayaka S K, Swasthika Jain T, Dr. Dayanand Lal

https://doi.org/10.30534/ijatcse/2020/72922020 